\title{
Présentation de l'édition Wahrnehmung und Aufmerksamkeit (Husserliana XXXVIII)
}

\section{Thomas Vongehr}

\section{(2) OpenEdition}

1 Journals

Édition électronique

URL : http://journals.openedition.org/alter/1517

DOI : $10.4000 /$ alter. 1517

ISSN : 2558-7927

Éditeur :

Association ALTER, Archives Husserl (CNRS-UMR 8547)

\section{Édition imprimée}

Date de publication : 1 octobre 2010

Pagination : $17-30$

ISBN : 2-9522374-6-8

ISSN : $1249-8947$

\section{Référence électronique}

Thomas Vongehr, «Présentation de l'édition Wahrnehmung und Aufmerksamkeit (Husserliana XXXVIII) », Alter [En ligne], 18 | 2010, mis en ligne le 01 juin 2020, consulté le 27 juin 2020. URL : http:// journals.openedition.org/alter/1517; DOI : https://doi.org/10.4000/alter.1517 


\section{PRÉSENTATION DE L'ÉDITION \\ WAHRNEHMUNG UND AUFMERKSAMKEIT \\ (HUSSERLIANA XXXVIII)}

Thomas Vongehr

\section{Introduction}

Avec mon ancienne collègue Regula Giuliani, j'ai commencé à travailler sur le thème de la perception et de l'attention à partir des manuscrits de Husserl en 1997 aux Archives Husserl de Fribourg. En 2004, la présente édition a été achevée aux Archives Husserl de Louvain, puis éditée dans les Husserliana à titre de volume XXXVIII. Je voudrais tout d'abord dire quelques mots sur la conception générale du Hua XXXVIII, puis donner un aperçu de la théorie husserlienne de la perception telle qu'elle s'est développée avant tout en lien avec le cours du semestre d'hiver 1904-1905, lequel est publié à titre de texte ${ }^{\circ} 1$ dans l'édition. Le fait que, à cet égard, je parle peu tout d'abord de la théorie husserlienne de l'attention tient à ceci (dont je m'expliquerai plus loin) que tous les manuscrits pertinents concernant la théorie de l'attention et présents dans le Nachlass n'ont pas encore été publiés.

\section{La conception de l'édition}

\footnotetext{
"L'entrelacement interne et sans doute aussi la nature des problèmes phénoménologiques fait qu'ils ne peuvent être résolus de façon isolée, et que l'on doit tantôt en développer une partie, tantôt une autre, pour que tout progrès accompli dans la clarification de l'une des parties jette en retour une lumière sur les autres » (Hua XXXVIII, p. 4, 1904-1905)
}

Initialement, l'édition devait comprendre, outre des parties du cours de 1904-1905 et des études plus anciennes qui lui sont étroitement liées (publiées dans l'édition en tant qu'appendices au texte $\mathrm{n}^{\circ} 1$ ), une partie du 
cours du semestre d'été 1909, qui traite également de la perception. Ce volume eût bénéficié d'un axe de gravité évident. Le cours de 1909 a alors été publié dans la série des Husserliana Materialien (Vol. VII). Aussi a-t-on choisi en vue de la publication, à la place du cours de 1909, d'autres textes qui témoignent de la préoccupation de Husserl pour les thèmes de la perception et de l'attention durant la période entre 1894 et 1912, à savoir peu avant la publication du volume I des Idées directrices.

Il est important d'indiquer que l'édition qui porte pour titre « Perception et attention » n'a pas la prétention (comme c'est le cas, par exemple, des volumes consacrés à l'intersubjectivité - Hua XIII-XV - et à l'imagination et à la conscience d'image - Hua XXIII) de publier tous les textes pertinents de Husserl sur ce thème. Ceci est particulièrement vrai dans le cas de la thématique de l'attention. De nombreux textes sur l'attention sont nés en 1911, c'est-à-dire durant la phase d'élaboration des Idées directrices, à savoir pendant le temps de réélaboration des Recherches logiques. Aucun de ces textes de recherche n'a été inséré dans l'édition (à l'exception du texte reproduit comme texte $n^{\circ} 4$ et intitulé « Directions de l'attention »). Husserl a consacré beaucoup de temps à ces manuscrits de recherche au milieu des années 1920, au moment où son assistant Ludwig Landgrebe les rassemblait avec des parties du cours de 1904-1905 sous le titre de travail : «Études sur la structure de la conscience », et les mettait au point en vue d'une publication. Cette publication n'a certes pas abouti, mais Landgrebe a utilisé des textes pour ce qui s'est appelé «Études logiques », lesquelles forment à leur tour la base du livre rédigé par Landgrebe et publié peu après la mort de Husserl : Expérience et jugement. L'ensemble du matériau manuscrit des «Études sur la structure de la conscience ", à savoir, également, les nombreux textes consacrés à l'attention, fait l'objet en ce moment à Louvain d'une mise au point par le professeur U. Melle et moi-même dans le cadre d'une édition qui comprend trois volumes. Mais il faudra sans doute compter encore une année au moins avant que ces volumes ne paraissent.

\section{Le cours du semestre d'hiver 1904-1905 : « Éléments principaux de la phénoménologie et de la théorie de la connaissance $»\left(\right.$ texte $n^{\circ} 1$ de l'édition)}

Le titre du volume édité se réfère directement aux parties publiées comme texte $n^{\circ} 1$ et issues du cours de Husserl du semestre d'hiver 1904-1905, lequel forme avec les appendices y afférents le texte le plus important de l'édition (230 pages) : d'où le fait, également, qu'il se trouve au centre des considérations qui vont suivre. Le cours de quatre heures du semestre d'hiver 1904-1905 que Husserl annonça sous le titre "Éléments principaux de la phénoménologie et de la théorie de la connaissance » est divisé en quatre parties (dans la forme qui nous est parvenue). Dans la première partie du cours, Husserl traite de la perception et, dans la deuxième partie, il traite du thème de 
l'activité de viser, de la visée, en l'occurrence, de l'attention et de l'intérêt. Ces deux parties sont publiées dans le volume XXXVIII. La troisième partie consacrée à l'imagination et aux actes de la conscience d'image est comme l'on sait publiée dans le volume XXIII. La dernière partie du cours de 19041905 traitait du temps. Cette partie a été mise au point en 1916 par l'assistante de Husserl, Edith Stein, puis publiée en 1928 par Martin Heidegger avec $\mathrm{d}$ 'autres manuscrits consacrés à la thématique du temps. Ce travail a été publié dans le volume $X$ des Husserliana. Malheureusement, on ne dispose pas encore, jusqu'à présent, de la version de cette quatrième partie du cours de 1904-1905 sous sa forme entièrement reconstruite, de sorte que ce cours important de 1904-1905 n'est malheureusement pas encore accessible dans sa totalité, c'est-à-dire sous la forme sous laquelle Husserl l'a prononcé.

Husserl raconte à son élève munichois, Johannes Daubert, qu'il s'efforce dans le cours de 1904-1905 « de sortir du champ des idées des Recherches logiques ». Cette remarque est extrêmement importante pour comprendre les intentions de Husserl à ce moment-là. En préparant son cours de 1904-1905, Husserl a recouru à "des études présumées prêtes pour la publication », lesquelles sont nées en 1898, à savoir peu de temps avant la publication des Recherches logiques, et qu'il avait vraisemblablement écrites à l'origine en vue $\mathrm{d}$ 'une suite à donner aux Recherches logiques. Alors que Husserl tentait dans les Recherches logiques une Introduction à la phénoménologie à partir de "l'expression et de la signification », il cherchait, avec les études de 1898 consacrées à la perception, à l'attention, à l'imagination, à ouvrir un chemin dans la phénoménologie qui soit, comme il le disait lui-même, "situé du côté de l'expérience et des données sensibles ». Dans le cours de 1904-1905, Husserl reprend en charge le chemin d'une théorie de l'expérience, à savoir l'analyse des « actes intellectuels simples, qui se situent à un niveau inférieur. Comme il apparaîtra plus clairement encore dans les années qui suivront, marquées par une confrontation avec Kant, en l'occurrence avec le néokantisme, son objectif est d'opérer une critique globale de la raison et, d'ailleurs, non plus seulement référée aux actes logiques intellectuels supérieurs dont il est question de façon centrale dans les Recherches logiques, mais aussi en relation avec les soi-disant actes inférieurs de l'expérience et de la sensibilité, à savoir les actes qui posent des valeurs. À la suite de Kant, il distingue trois strates de conscience, qui sont fondées les unes sur les autres; la représentation et le jugement en tant que domaines de la raison théorique, puis la strate des sentiments dotés de valeur, qui est à son tour fondée dessus, et enfin la strate qui est à son tour fondée dessus, celle des actes qui sont des actions, en tant que sphère de la raison pratique. Dans ses nombreux cours à Göttingen, Husserl s'efforce d'analyser de façon concrète ces différents actes et strates de conscience (par exemple dans le cours Chose et espace de 1906 (Hua XVI), dans le cours sur la signification de 1908 (Hua XXVI), dans le cours sur la perception de 1909 (Hua VII) et puis, pour ce qui concerne les actes qui posent des valeurs, dans le cours sur l'éthique de 1909 (Hua XXVIII). 


\title{
L'attention
}

Par contraste avec les première et troisième parties du cours de 1904-1905 (à savoir les parties consacrées à la perception et à l'imagination), où Husserl a réélaboré de nombreux passages des anciennes études de 1898, la deuxième partie du cours sur L'attention et la visée, à l'exception d'une partie introductive et d'une partie conclusive qui propose une rapide évaluation, repose directement sur son élaboration du thème de l'attention datant de 1898. Ainsi qu'il l'explique à ses étudiants, Husserl a lu cet essai, à l'exception de quelques «excursus dans le domaine psychologique». Ses remarques marginales montrent que Husserl, à ce point, n'était déjà plus à proprement parler satisfait de son ancienne présentation. Vraisemblablement, Husserl manqua de temps, lors de la préparation du cours, pour se centrer de façon précise sur la thématique de l'attention, c'est-à-dire pour la mettre au niveau des analyses de 1904-1905.

En résumé : le cours de 1904-1905 est important dans la mesure où Husserl tente d'y dégager un nouveau champ de recherches pour la phénoménologie, en relation avec une théorie de l'expérience, à savoir une critique globale de la raison au sens kantien par laquelle l'introduction gnoséologique ou logique des Recherches logiques devrait être dépassée. Assurément, Husserl n'a pas toujours réussi à réélaborer d'anciennes études antérieures aux Recherches logiques pour les insérer dans ce cours, ce qui a conduit à certaines incohérences et à certaines discontinuités dans le texte. En comparaison avec les développements plus tardifs consacrés à la perception que l'on trouve par exemple également dans l'«Ausarbeitung sur la perception » de 1912 imprimé dans l'édition à titre de texte $n^{\circ} 3$, on ne peut que critiquer la perspective limitée où se meut l'investigation de 19041905, étant donné que Husserl n'y thématise ni la question de la relation des perceptions avec le Je de la perception, ni celle de leurs relations avec les kinesthèses, ni non plus corrélativement, ne traite-t-il de la signification de la chair pour la perception. De surcroît, il manque aussi, au regard de la phénoménologie plus tardive, la prise en considération à mesure si importante de la réduction phénoménologique, que Husserl n'introduit qu'environ un an plus tard après le cours de 1904-1905. Aussi n'est-il pas étonnant que Husserl ait été le plus souvent, dans les années suivantes, à l'égard de ce cours de 1904-1905.

\section{La théorie husserlienne de la perception}

\author{
Les contenus présentants, les sensations, sont animés par le caractère de l'appréhension, \\ et cela constitue l'apparaître de l'objet. (Hua XXXVII, p. 27, 1904-1905) \\ La même chair qui me sert de moyen pour toute perception, est pour elle-même un \\ empêchement lorsqu'il y a perception, et est une chose remarquablement imparfaite. \\ (Hua IV, p. 159, env. 1913)
}

La perception est pour Husserl une « conscience originale », à savoir « le mode originaire de l'intuition » et, par là, un vécu de conscience tout à fait 
remarquable. Lorsque nous percevons quelque chose, notre visée est de saisir l'objet «sur un mode originaire », dans son ipséité « charnelle ». Normalement, nous ne doutons pas de ce que nous percevons. Dans la pratique quotidienne de la vie, la perception nous garantit sa certitude d'être, que Husserl saisit dans les Idées directrices I par le concept de "thèse générale de l'attitude naturelle ». Selon la terminologie phénoménologique, l'activité de percevoir est un acte de présentation intuitive, que l'on doit distinguer d'autres modes de l'intuition comme l'imagination, le souvenir, l'attente ou la conscience d'image, lesquels ne prétendent pas, en tant que présentifications intuitives, fournir une auto-donation incarnée de l'objet présumé.

La théorie phénoménologique de la perception, formulée pour la première fois dans les Recherches logiques, est inspirée par une critique exercée à l'encontre de l'interprétation de la perception comme théorie des signes tout autant que comme théorie des images. Cette dernière est avant tout présente dans l'école très influente de Helmholtz. Par contre, la critique par Husserl de la théorie de la perception comme image-copie prend sa source dans la question énigmatique concernant le statut ontologique de l'objet intentionnel, posée notamment par Twardowski et discutée dans l'école de Brentano. Comment se peut-il que nous puissions viser des objets en nous les représentant, ces objets pouvant ne pas exister du tout, comme par exemple des cercles carrés, etc. ? S'il n'y a là, dans ce cas, qu'une image intramentale de l'objet extérieurement non existant, est-ce que, dans le cas d'objets réellement existants, des images-copies intentionnelles mentales sont présentes en sus à côté d'eux?

Mais l'influence de Husserl s'étend au-delà de la psychologie descriptive de l'école de Brentano, dans le cadre de laquelle Husserl a également rencontré la distinction entre les représentations propres (intuitives) et impropres (symboliques), distinction importante pour ses analyses de la perception. La confrontation avec la physiologie des sens mise en avant par Helmholtz et avec l'école de Hering joue ici un rôle aussi important que, par exemple, également, la confrontation avec la théorie de la sensation de Mach. Dès ses premiers travaux de l'époque antérieure aux Recherches logiques, Husserl s'est attelé à des problèmes concernant l'espace et, en lien avec le plus ancien élève de Brentano, Stumpf, à l'origine psychologique de la représentation de l'espace. Il revient plus tard, à nouveau, sur les thèmes qui y sont traités : la perception en profondeur de la vision, la détermination de l'espace visuel et de l'espace tactile, de la corrélation entre le mouvement des organes des sens (les kinesthèses) et le développement de la représentation de l'espace. Même les investigations plus tardives de William James, de Theodor Lipps et d'Alexander Pfänder consacrées à la problématique de la perception et de l'attention ont influencé Husserl, ce pour quoi les travaux de Pfänder et Lipps devraient être également pris en considération en tant qu'investigations proprement phénoménologiques. 


\section{Le rôle de la sensation et de la perception}

La théorie de la perception fondée dans les Recherches logiques, dans les Leçons de 1904-1905 et dans les Idées directrices I est en accord, plus avant, avec la détermination du rôle que jouent les sensations dans la perception. Les data de sensations, désignés dans la terminologie des Recherches logiques sous l'expression de " contenus primaires", dont font partie " certains vécus "sensuels" - unitaires conformément au genre supérieur -, "des contenus de sensation" comme les data de couleur, les data tactiles, sonores, etc. » ne possèdent encore rien, en eux-mêmes, de l'intentionnalité. L'appréhension des sensations en tant que telles n'est mise en place, si l'on sui Husserl, qu'à travers une " couche donatrice de sens ", à travers des caractères d'acte, de sorte que, au sein du flux des vécus de la perception, il convient de distinguer entre "une couche matérielle et une couche noétique». La sensation momentanée de couleur est ainsi explicitement distinguée de la couleur de la chose qui se présente en elle, laquelle est attribuée à la chose en tant que qualité. Voici donc ce qui est énoncé de la plus façon la plus claire dans le cours de 1904-1905 relativement à l'opération d'appréhension de la conscience: «Les contenus présentants, les sensations, sont animés par le caractère de l'appréhension, et cela constitue l'apparaître de l'objet » (Hua XXXVIII, p. 27). La morphè intentionnelle est opposée à la hylè sensuelle ; en même temps, «la dimension remarquable du dédoublement et de l'unité » des deux moments est soulignée. On a critiqué une telle théorie de la perception orientée par le "schéma du contenu d'appréhension et de l'appréhension », en raison de sa proximité avec le sensualisme et de sa tendance au dualisme (sensation-perception).

En dépit d'intuitions fondatrices, que Husserl a conquises en particulier à partir d'analyses consacrées au temps, à la phénoménologie génétique et aussi à travers l'introduction des recherches noématiques, c'est-à-dire non dirigées vers les opérations d'actes, il n'a jamais pris clairement congé de cette conception de la sensation. C'est pourquoi, le concept de kinesthèse, introduit dans sa théorie de la perception quelque deux années seulement après le cours de 1904-1905, rend nécessaire une nouvelle détermination de la distinction traditionnelle entre la matière et la forme du vécu. Les sensations ne sont plus des impressions données de façon purement passive, elles dépendent de ma mise en mouvement charnelle. Les kinesthèses en tant que sensations de mouvement, par exemple de l'œil ou de la tête, sont au fondement d'un « double jeu constitutif » de la pure sensation en tant que datum et de la sensation de la position. Cette dernière est responsable de la localisation spatiale de l'objet entendu comme chose. Les kinesthèses ne doivent donc pas être pensées sans une chair qui ressent. La chair, à laquelle Husserl a consacré des analyses denses d'un point de vue descriptif dans les Idées directrices II publiées de façon posthume, est le «point zéro » de l'orientation, puisque tous les points dans l'espace reçoivent leur détermination de lieu (audessus, en dessous, loin, proche) de la position elle-même dans l'ici. 


\section{La perception comme thème phénoménologique universel}

La préférence accordée par Husserl au sens optique a conduit à une détermination de la perception visuelle de la chose élaborée conséquemment de façon extensive en tant que mode remarquable de la conscience intentionnelle ; assurément, d'autres sens perceptifs, comme par exemple le sens tactile, le sens auditif ou le sens kinesthésique déjà mentionné, se trouvent également pris en considération dans une mesure plus limitée. Dans un appendice au cours de 1904-1905, se trouve même abordé, quoique brièvement, le phénomène perceptif complexe de la synesthèse (p. 208 sq.), comme dans le cas du velours, qui peut provoquer une sensation tactile sur la base de la « vision » de sa douceur. À l'amplitude du concept de perception en tant que conscience de la donation en personne correspond une multiplicité de types de perceptions auxquels Husserl a consacré de vastes recherches: perceptions interne et externe, immanente et transcendante, adéquate et inadéquate, sensible et non sensible (catégoriale), de la chose et de l'espace, du moi et de la chair, de l'étranger (empathie), du temps ou encore de l'essence. À cet égard, toutes ces recherches phénoménologiques consacrées à la perception sont unifiées par l'effort de Husserl de rendre intelligible la compréhension des actes de degré supérieur, à proprement parler intellectuels (comme les actes de jugement, de raisonnement, etc.), à travers une investigation de ces «actes » dits «simples, situés en dessous des actes intellectuels", au sens d'une théorie de l'expérience. Tel est également l'horizon gnoséologique à partir duquel les actes de la perception sont thématisés dans les Recherches logiques. Finalement, Husserl tente également, dans l'œuvre tardive qui porte sur des questions logiques, notamment dans Expérience et jugement, de déterminer la perception dans son rôle fondateur comme évidence antéprédicative en vue des opérations idéalisatrices de la logique. Même dans la phénoménologie génétique, qui se développe dans les années 1920, Husserl s'efforce de procéder à une élucidation de la synthèse passive en tant que soubassement de tous les actes perceptifs, ce pour quoi le concept de l'association, au sens d'une formation transitoire de champs sensibles, lui sert ici de fil conducteur. De façon intéressante, dans ces analyses génétiques consacrées à la synthèse passive, Husserl, mettant à profit une plus grande maturité phénoménologique, recourt à des recherches et à des intuitions qui datent principalement de la période précédant les Recherches logiques et qui renvoient à la tradition de l'école de Brentano. Dans les années 1930, sous le signe du «monde de la vie » de la Krisis et des manuscrits de recherche y afférents, il mène une recherche consacrée au lien de dépendance entre l'expérience du monde de la vie et la science objective. Ces recherches se fondent essentiellement sur les analyses des actes de représentation intuitive en tant que modalités de l'accès primaire au monde. 


\title{
Les analyses concrètes de la perception peuvent-elles exercer une fascination?
}

\author{
Même si je ne vois pas à proprement parler le côté arrière de l'objet, il fait pourtant \\ partie de lui comme objet que je perçois. Comment le sens, la visée de ma perception \\ peuvent-ils se rapporter à lui ? (Hua XXXVIII, p. 35, 1904-1905) \\ La perception externe prétend constamment accomplir quelque chose qu'elle est, de \\ par son essence propre, hors d'état d'accomplir. Aussi contient-elle d'une certaine \\ manière en elle-même une contradiction. (Hua IX, p. 3, 1920-1921)
}

Rappelons à présent, le point de départ des analyses husserliennes consacrées à la perception. On sait que, dans la perception actuelle, il n'y a jamais qu'un seul côté de la chose qui est «à proprement parler »vu, tandis que, par exemple, le côté arrière est seulement co-visé «sur un mode impropre». Exemple standard: je vois la table là-bas devant moi. Cela signifie bel et bien : je prétends voir l'ensemble de la table. Toutefois, je dois dire, en analysant les choses de plus près que, à partir de mon point de vue actuel, je ne peux voir que la partie avant de la table, peut-être aussi une partie du côté, mais pas, par exemple, le côté arrière de la table, qui est à présent caché pour moi. Pour désigner ce mode de donation nécessairement perspectiviste de l'objet de la perception, Husserl utilise dans ses textes plus tardifs le terme connu de « profil ». Je peux laisser mon attention errer pour ainsi dire à son gré. Je ne peux voir le côté arrière de la table que si je quitte mon point de vue actuel, que si, par conséquent, je change ma perspective, que je me dirige vers la table, que je tourne autour d'elle, pour regarder le côté arrière. Mais, alors, c'est ce qui était le côté avant de la table qui est devenu un côté arrière caché, etc. Par conséquent, jamais je ne vois la chose «table» simultanément dans tous ses aspects possibles. Aussi, si l'on suit Husserl, la perception contient-elle dans son essence un paradoxe, une contradiction, ainsi qu'il le formule de façon prégnante dès la première phrase de son cours des années 1920 (publié sous le titre «De la synthèse passive » dans le Hua XI) : «La perception externe prétend constamment accomplir quelque chose qu'elle est, de par son essence propre, hors d'état d'accomplir. Aussi contient-elle d'une certaine manière en elle-même une contradiction. » (Hua IX, p. 3, 1920-1921) Formulé de façon positive, cela signifie que, quoique tous les aspects et les côtés de la chose spatiale ne peuvent jamais être perçus simultanément, nous prétendons toutefois voir la chose elle-même.

Aussi triviale que cette observation puisse paraître, Husserl a toujours été fasciné par cette contradiction inhérente à la perception. D'autres philosophes ont également subi cette fascination. Ainsi, Sartre s'est extasié que l'on puisse observer un verre de cocktail et que sa description relève de la philosophie, et dans son roman, La Nausée, le protagoniste ne peut détacher son regard de la racine du marronnier, qu'il ne peut s'empêcher de percevoir. 
Or, le problème se pose pour Husserl, dans ses analyses de la perception, de savoir comment cet être-codonné de côtés arrière non intuitifs, etc. doit être explicité phénoménologiquement. La question qui pose problème est la suivante: comment cette visée, cette conviction de l'être-donné de l'ensemble de l'objet perceptif advient-elle? À propos du côté avant, qui fait l'objet d'une vision actuelle (proprement dite), il est par exemple facile de dire que j'avais des sensations déterminées qui sous-tendent la perception et rendent possible l'appréhension, etc. Il en va autrement de l'appréhension du côté arrière. Je ne la vois pas là à proprement parler, aussi ne puis-je avoir des sensations qui pourraient motiver une telle vision. Les côtés actuellement non visibles de la chose, qui sont toujours parties prenantes de la perception dans son ensemble, ne peuvent alors, si l'on suit Husserl, être donnés à travers des représentations de l'imagination, puisque cela aurait pour conséquence de brouiller la distinction entre l'être-donné charnel et l'être-donné imagé. Husserl a envisagé différentes solutions possibles. Dans le cours de 1904-1905, prenant appui sur les Recherches logiques, il utilise le schéma de la ressemblance et de la contiguïté, qu'il abandonnera cependant plus tard, parce qu'il engendre l'illusion que la perception d'une chose spatiale serait à comprendre sur le modèle des significations langagières. Et, en effet, Husserl parle d'un caractère de renvoi ou de signe, qui a partie liée avec la détermination présentée à proprement parler, relativement à ce qui apparaît de façon impropre. Pour décrire ce lien, Husserl utilise le terme de " contiguïté », employé dans la psychologie de l'association du XIXe siècle.

Dans les Idées directrices I, Husserl introduit alors le concept d'horizon, de façon à clarifier cette opération d'apprésentation en tant qu'être-codonné $\mathrm{d}^{\prime}$ un non-donné à proprement parler et, d'une certaine manière, pour réviser sa théorie initiale de la perception. Dans le cas de la perception, « un noyau "effectivement présenté" est entouré sur un mode apprésentatif par un horizon de "codonnées" impropres et d'indétermination plus ou moins vague» (Hua III/1, p. 91). Ainsi, le côté non vu de la chose n'est jamais entièrement indéterminée, étant donné qu'elle est pré-esquissée sur un mode typique par le côté qui est vu, c'est-à-dire que nous "savons » que le côté arrière de la table est également coloré, doté d'une forme corporelle, etc. : "Un tel halo de conscience trouve son sens, malgré sa vacuité, dans la forme d'une esquisse préalable, laquelle prescrit une règle à ce passage à de nouvelles apparitions s'actualisant» (Hua XI, p. 6). De cette manière, les horizons sont des « potentialités pré-esquissées », notamment de déterminations plus précises se développant indéfiniment (par exemple, la table, telle qu'elle apparaît depuis son côté arrière, telle qu'elle apparaît de l'intérieur d'elle-même etc.), mais ils ne peuvent jamais atteindre leur objectif d'une donation parfaite de la chose. La donation parfaite de la chose est, suivant en cela la théorie husserlienne plus tardive de la perception (pour ainsi dire durant la période des Idées directrices I en 1912), une idée au sens kantien. La perception est caractérisée par des anticipations motivées (des attentes et des protentions) qui saisissent par avance ce qui peut se manifester dans le futur. 


\title{
L'attention
}

De telles pré-saisies ne sont pas toujours remplies, mais peuvent également conduire à la déception ou au conflit (contre toute attente, la forme sphérique est cabossée derrière). Ainsi, le processus perceptif est un "processus de prise de connaissance permanente » dont l'inscription est téléologique, «qui conserve comme signifiant ce qui est connu et crée ainsi un sens qui se transforme toujours et qui s'enrichit toujours plus » (Hua XI, p. 12). La prise de connaissance n'est pas seulement une chose instantanée, mais elle conduit à des instaurations durables de sens et à des acquisitions de sens (à des habitualités et à des sédimentations), auxquelles l'individu et la communauté peuvent toujours à nouveau revenir.

\section{À quoi sommes-nous attentifs lorsque nous accomplissons des actes intentionnels? Que visons-nous?}

\begin{abstract}
Se référer en visant, ce dont nous parlons ici, est en relation apparemment étroite avec le discours sur l'attention. Celle-ci est à l'évidence tout à fait plurivoque, et il $n^{\prime} e s t$ pas aisé de circonscrire clairement les concepts qui lui sont étroitement liés et se rattachent à elles. (Hua XXXVIII, p. 86, 1904-1905)

Il est évident que la possibilité idéale d'accomplir la perception partielle et, en même temps, la possibilité idéale d'accomplir l'identification sur le mode de l'évidence trouve son fondement dans l'essence de la perception globale donnée (l'apparition globale), ce qui place l'une et l'autre en relation de tout et de partie. » (Hua XXXVIII, p. 33)
\end{abstract}

On peut à présent se demander comment la thématique de l'attention s'articule avec la première partie du cours consacré à la perception. Dans ses recherches sur la perception, Husserl met toujours à nouveau en avant la distinction entre l'avant-plan et l'arrière-plan du perçu, entre le perçu à proprement parler et le perçu qui ne l'est pas. Dans des manuscrits plus tardifs, la possibilité de faire ressortir, dans les perceptions partielles, de nouveaux aspects à même ce qui apparaît à proprement parler, en l'occurrence, de transformer l'arrière-plan qui n'est pas à proprement parler vu en un avant-plan actuel etc. est déterminée comme une liberté essentielle du sujet et caractérisée par l'expression «Je peux». Cela signifie la chose suivante : «je peux » changer mon point de vue présent et, moyennant une nouvelle perception, transformer le perçu préalablement impropre en quelque chose de perçu à proprement parler, etc. Par le changement possible de perspective, nous dirigeons notre attention sur de nouveaux aspects propres à l'objet, qui n'étaient pas au préalable clairement saisis. La possibilité du «Je peux » appartient donc à l'essence de la perception, même lorsque, fréquemment, des limites sont posées à l'exécution de fait du changement de perspective, car je ne peux pas tourner autour de tous les objets pour parvenir à regarder son côté arrière. Relativement à cela se pose 
à Husserl une série de questions nouvelles, qu'il rattache principalement, dans le cours, au concept de la visée. Ses questions touchent principalement les opérations de synthèse et la détermination de la relation entre la multiplicité et l'unité, en l'occurrence les touts et les parties de la perception. Concrètement, Husserl demande comment il est possible que les multiples aperçus de l'objet, que nous pouvons prendre en vue dans la pratique perceptive quotidienne, puis, avant tout, lors d'une variation de notre attention conduite de façon méthodique, puissent garantir l'identité de l'objet perçu. Pour poursuivre avec cet exemple perceptif simple, nous considérons l'objet « table » de différents côtés. La question de Husserl est la suivante : qu'est-ce qui fait l'unité de notre visée, à savoir, qu'est-ce qui fait que nous visons un objet, nommé «table », donc un seul et même objet, et non une pluralité d'objets différents, qui se manifestent à nous dans le profilage perspectiviste?

Partant de cette problématique développée à partir de la théorie de la perception, et qui pose la question de la relation entre la multiplicité perceptive et l'unité de la visée, Husserl passe ensuite, dans son cours, à son étude de 1898 consacrée à l'attention. Cela conduit à mon sens à une rupture, étant donné que l'attention, dans l'étude plus ancienne, est abordée dans un contexte que l'on peut désigner davantage comme relevant de la disposition psychologique. Assurément, on rencontre fréquemment chez Husserl un semblable positionnement bipartite dans la thématisation de l'attention. D'un côté, son intérêt se porte vers l'élucidation phénoménologique de l'intérêt et de l'attention, au sens d'une clarification de la question de savoir comment - considérée sous un jour passif ou actif - l'affection, l'attraction, le fait d'être attiré par quelque chose, ou bien de se tourner (en l'occurrence, de se détourner) vers quelque chose, qui est propre à l'attention doit être expliqué. Une recherche à laquelle Husserl se livre par exemple également dans les cours génétiques des années 1920. Un exemple typique de cette orientation de la recherche est proposé avec son analyse du sifflement strident du train, que nous entendons alors que nous sommes sur le quai (voir par exemple Hua XXXVIII, p. 93). Si le sifflement dépasse une certaine intensité sonore (ce qui dépend à chaque fois de la disposition propre), nous ne pouvons nous arracher à ce sifflement. Si l'intensité sonore s'avère moins élevée et si, éventuellement, une conversation nous retient de surcroît, qui nous captive, nous ne serons absolument pas attentifs au sifflement, ou bien il restera l'arrière-plan de notre attention, sans que nous dirigions véritablement notre attention vers lui. Nous trouvons ainsi dans les écrits de Husserl de nombreuses analyses similaires d'une grande densité en matière de description phénoménologique, par exemple dans les textes de l'édition où il examine les phénomènes du relief attentionnel ou du rythme de la tension et de la détente. D'un autre côté cependant, les recherches consacrées à l'attention vont dans un sens qui se rapporte davantage à la conscience de la signification et, par là, à la structure de la visée, ainsi qu'aux structures prédicatives, verbales de la langue. De façon générale, on peut 


\section{L'attention}

constater qu'une palette largement diversifiée d'intérêts de recherche se cache chez Husserl derrière le thème de l'attention, en relation avec lesquels il utilise de façon variée les expressions de "visée », « intérêt ", "remarquer », " porter son attention», "se tourner vers », "être-dirigé vers », "thème», « représentation», «prise de position », «tendance», « explication » etc. Dans la sphère du langage elle-même, il y a quelque chose comme un arrière-plan et un avant-plan (thématique) et, par là, également, un changement possible de l'orientation de la visée, à savoir de l'attention. Dans son cours consacré à la théorie de la signification de 1908 (Hua XXVI), Husserl développe un nouveau concept de signification, qui donne une indication de ce que sera le concept noématique de signification présenté pour la première dans les Idées directrices I en 1913, et qui est développé en 1908 à partir de la conscience thématique de la signification, c'est-à-dire à partir de la question, que l'on peut restituer ici en la simplifiant, de savoir à quoi nous sommes thématiquement attentifs lorsque nous utilisons des expressions déterminées. Clarifions pour nous les parallèles qui existent entre le rôle que joue l'attention dans la conscience de perception et dans la conscience de signification et, par là, également, le problème de la relation entre l'unité et la pluralité (le sens et la signification) qui surgit dans la conscience de la signification, à partir de l'exemple suivant (bien connu) (voir en particulier Hua XXVI, § 8), sans pour autant pouvoir rentrer dans les détails : j'emploie l'expression "le vainqueur de Iéna ». Vers quoi suis-je orienté ? Quel est mon thème ? On répondra peut-être : je veux parler de Napoléon. Mais Husserl constate que je puis bien également mentionner Napoléon en utilisant l'expression "le perdant de Waterloo », et que, en même temps, les deux expressions («le vainqueur de Iéna », «le perdant de Waterloo ») désignent justement aussi quelque chose de différent. Plus avant, Husserl pose la question de savoir comment une théorie de la signification peut faire droit à quelque chose que je puis identifier sous un seul et même objet de référence tout en le visant à travers une multiplicité d'expressions différentes.

\section{Essais de phénoménologie de l'attention}

À côté < d'une phénoménologie de la perception, de l'imagination, du temps, de la chose $\gg$, j'ai aussi tenté une phénoménologie de l'attention... (Note de Husserl du 25 septembre 1906 dans son Journal, Husserl-Chronik, p. 99)

Or, on doit bien considérer ce point parmi les plus fondamentaux de la théorie de l'attention, lequel est constamment passé sous silence, que le fait de se tourner vers l'objet ne signifie pas que l'on se tourne vers les contenus de la conscience... (Hua XVI, p. 20, 1908)

L'attention entendue comme un "se diriger-vers ", lequel peut se produire avec un "vécu intentionnel» qui ne le contient pas, n'est pas un "acte de degré supérieur", "n'est pas une conscience fondée ", n'est pas un deuxième acte de "se diriger-vers " pour ainsi dire sur la base d'une apparition perceptive (sans un «se diriger-vers »). Certainement, il y a quelque chose de vrai dans une telle façon de 
parler, et je l'ai déjà reconnu dans les Recherches logiques. Pourtant, une clarté analytique plus grande est ici requise. On a besoin de concepts justes $d^{\prime}$ " acte », de "conscience». <...> En tout cas, on ne peut pas employer la façon de parler suivante: "le se diriger-vers n'est pas un acte", "l'attention est un mode d'acte, mais pas un acte propre de degré supérieur " etc., sans avoir au préalable clarifié tous les concepts y afférents (Manuscrit inédit qui fait partie des "Études sur les structures de la conscience », A VI 8 I/157a, sans doute 1911)

Dans la deuxième moitié du XIXe siècle - motivé également par les possibilités techniques croissantes de la psychologie expérimentale - est apparu chez les philosophes/psychologues un intérêt marqué pour la thématique de l'attention. Husserl connaissait assez bien les recherches correspondantes, comme l'attestent par exemple ses prises de position par rapport à Wundt, Brentano, Herbart, Höfler, Hamilton, Stumpf, James, entre autres, dans les textes de l'édition. Ainsi il qualifie par exemple la théorie de l'attention développée par Stumpf dans sa Psychologie du son (1883) $\mathrm{d}^{\prime}$ « analyses magistrales » (Hua XXII, p. 293), même si la définition donnée par Stumpf de l'intérêt comme un "plaisir pris à remarquer », ainsi que l'on peut la retrouver dans le manuscrit publié dans l'édition à titre d'appendice II, est explicitement critiquée. Dans l'étude de 1898 sur l'attention qui est reproduite dans l'édition, Husserl remarque qu'il a été tenté plus tôt d'identifier l'intérêt et l'intention percevante (Hua XXXVIII, p. 106). C'est vraisemblablement la confrontation à ce propos avec les écrits de James qui a eu pour effet d'interrompre la poursuite prévue des «Études psychologiques sur la logique élémentaire» parues en 1894 (Ниа XXII), où Husserl a défendu une telle position. Dès son écrit d'habilitation Sur le concept de nombre en 1887, dans lequel Husserl mène un examen de l'origine du concept de nombre qu'il qualifie encore de psychologique, le concept d'attention joue un rôle, concernant par exemple la question de savoir comment des représentations isolées peuvent être unifiées sous un concept unique (voir Ниа XII, p. 333). Toutefois, ce sont les confrontations conduites dans la deuxième Recherche logique avec les théories de l'abstraction qui ont cours alors, à savoir les théories de la saisie du général, qui fondent les déterminations ultérieures de l'attention. La critique de Husserl, qu'il résume au paragraphe 22 de la deuxième Recherches logiques sous le titre « Déficits fondamentaux de l'analyse phénoménologique de l'attention ", s'ordonne autour du fait que l'attention fait l'objet dans ces théories d'une interprétation fallacieuse : elle serait un acte dirigé vers des contenus vécus ou vers des sensations. Plus tard, Husserl parle de mutations attentionnelles ou de modifications des actes intentionnels ou des unités intentionnelles, et tente par là, semble-t-il, d'éviter à propos de l'attention d'utiliser le concept d'acte. À plusieurs reprises, Husserl indique la nécessité d'une phénoménologie de l'attention, qu'il n'a certes jamais développée lui-même de façon systématique. Ses diverses recherches sur l'attention dans les années de Göttingen trouvent un premier résultat dans le $\S 92$ des Idées directrices I, paragraphe très dense et de ce fait peu aisément compréhensible. Au centre de ce paragraphe se 


\section{L'attention}

trouve la théorie des mutations attentionnelles, qui fait déjà l'objet d'une discussion dans les appendices de l'édition ( $\mathrm{n}^{\circ}$ XVII, XVIII) datant de 1909. L'attention est une "mutation attentionnelle » d'unités déjà constituées sur un mode intentionnel, explique Husserl dans les Idées directrices I. L'attention est une «forme fondamentale de modification intentionnelle » et, plus avant, on trouve dans un autre texte la détermination suivante: " L'attention n'est pas une intention » (Hua XXIV, p. 250). L'accomplissement de l'attention présuppose l'intentionnalité. Husserl critique le fait que, même si l'attention est "un thème central de la psychologie moderne ", il n'en reste pas moins que «la relation d'essence entre l'attention et l'intentionnalité » abordée dans les Recherches logiques (Hua III, 1, p. 215 Rem. 1) n'a pas été prise en considération. Seules les recherches de Stumpf et de Lipps font l'objet d'une appréciation positive. Dans son œuvre tardive, Husserl, influencé par son examen de l'opération de la synthèse passive, tend toujours à nouveau à décrire l'attention à son niveau le plus bas comme une réceptivité (un éveil affectif), c'est-à-dire comme une tendance du moi dirigée vers un stimulus qui affecte le moi. Ainsi, l'attention est décrite dans Expérience et jugement comme une «tendance du moi <..> dirigée vers un objet intentionnel » (Expérience et jugement, p. 85). Par là, une nette dominante des recherches de Husserl sur l'attention se trouve ici minorée : en incluant le Je dans l'analyse phénoménologique, ce qui se dessine à peu près depuis 1905 - dans le cours de 1904-1905, il n'en est pas encore question, pas plus que dans les Recherches logiques -, le vécu de conscience a pour ainsi dire admis un centre, c'est-à-dire que l'acte de conscience possède un point de rayonnement. À partir de là, on trouve toujours à nouveau dans la théorie husserlienne de l'attention l'image du « rayon visuel du Je » ou du « rayon attentionnel »; Husserl aime aussi à employer la métaphore que l'on trouve chez Lotze du "regard qui erre", et ce, bien qu'il ait encore indiqué dans les Recherches logiques que l'attention ne saurait être simplement envisagée comme « une fonction clarificatrice et focalisatrice " (Recherches logiques, p. 164). Opérant avec de telles métaphores, la théorie de l'attention possède, en comparaison avec les recherches dans l'œuvre tardive, une dimension plus nettement volontaire, et a de ce point de vue l'objet de critiques diverses, émanant par exemple de Gurwitsch, mais aussi de Stumpf, qui doutait «que la théorie des rayons de Husserl procure une vue plus profonde de l'essence de l'attention. $<\ldots>$ Ce ne sont pourtant que des images, des pierres à la place du pain de la connaissance véritablement élucidante».

Thomas Vongehr

Husserl Archiv Leuven 